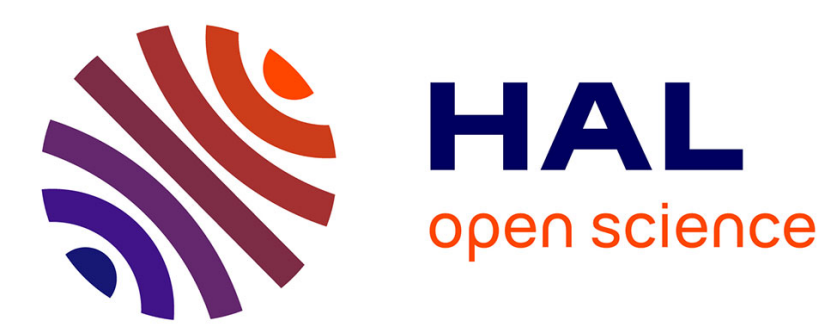

\title{
Cognitive function assessment for personalized heart failure disease management programmes
}

\author{
João Pedro Ferreira
}

\section{To cite this version:}

João Pedro Ferreira. Cognitive function assessment for personalized heart failure disease management programmes. European Journal of Heart Failure, 2021, 23 (7), pp.1215-1216. 10.1002/ejhf.2183 . hal-03469216

\section{HAL Id: hal-03469216 \\ https://hal.univ-lorraine.fr/hal-03469216}

Submitted on 7 Dec 2021

HAL is a multi-disciplinary open access archive for the deposit and dissemination of scientific research documents, whether they are published or not. The documents may come from teaching and research institutions in France or abroad, or from public or private research centers.
L'archive ouverte pluridisciplinaire HAL, est destinée au dépôt et à la diffusion de documents scientifiques de niveau recherche, publiés ou non, émanant des établissements d'enseignement et de recherche français ou étrangers, des laboratoires publics ou privés. 
Cognitive function assessment for personalized HF disease management programmes

João Pedro Ferreira, MD, $\mathrm{PhD}^{1,2}$

${ }^{1}$ Université de Lorraine, Inserm, Centre d'Investigations Cliniques Plurithématique 1433, and Inserm U1116, CHRU, F-CRIN INI-CRCT (Cardiovascular and Renal Clinical Trialists), Nancy, France.

${ }^{2}$ Cardiovascular Research and Development Center, Department of Surgery and Physiology, Faculty of Medicine of the University of Porto, Porto, Portugal.

Contact to:

Dr João Pedro Ferreira

Centre d'Investigation Clinique 1433 module Plurithématique

CHRU Nancy - Hopitaux de Brabois, Institut Lorrain du Coeur et des Vaisseaux Louis Mathieu

4 rue du Morvan, 54500 Vandoeuvre les Nancy

Tel : +33 (0) 383157315

Fax : +33 (0) 383157324

Mail: j.ferreira@chru-nancy.fr 
Cognitive impairment is prevalent among patients with heart failure (HF) and impacts long-term outcomes. ${ }^{1}$ The reduction in cerebral perfusion, microvascular damage and cerebral infarcts, decreased cardiac output and low blood pressure, and alterations in cerebrovascular reactivity may trigger and aggravate the cognitive alterations in HF patients. ${ }^{2}$ Compared to HF patients without cognitive impairment or dementia, those with cognitive alterations are more likely to be readmitted and to die from any cause. ${ }^{3}$ An interplay of several factors contributes to the poor outcome of HF patients with cognitive impairment, including the deterioration in the ability for self-care, the need for social, third-person or institutional support, the incapacity of adhering to treatment regimens (especially complex regimens with multiple pills), the difficulty in describing symptoms and changing health behaviours. ${ }^{4}$ Despite the growing knowledge about the impact of cognitive alterations in HF, little is known on how to improve cognitive function and how cognitive alterations may impact specific HF interventions, including HF management programs.

In this issue of the journal Huynh QL and colleagues present an interesting analysis aiming to determine if the degree of cognitive impairment could influence the response to a HF management program. From a total pool of 1152 patients that had been prospectively followed for 1 year, 324 (28\%) received a HF disease management program (HF-DMP) with a 1-month duration that included postdischarge phone calls and home visits, therapeutic and self-care education, transition of care (with a "transition" coach), exercise guidance and clinical review/assessment. As the HF-DMP was not allocated randomly, in order to mitigate the imbalance between patients who received and not received the program, the 324 patients who had received the HF-DMP were "matched" (using a propensity-score matching adjustment technique) with 648 patients who did not receive the HF-DMP 
(i.e., they received "usual care"). At the time of study enrollment (before hospital discharge) the patients were screened for cognitive impairment using the Montreal Cognitive Assessment (MoCA) and during the 1-year follow-up hospital readmissions, deaths and days-at-home were recorded. Confirming prior studies, ${ }^{5}$ poorer cognitive function was prevalent and independently associated with worse outcomes. On the other hand, the HF-DMP was independently associated with better outcomes, a finding that had also been previously documented. ${ }^{6}$ However, whether the efficiency of a HF-DMP could be influenced by patients' cognitive function has not been fully explored and is an important research question.

Based on the findings of this study, patients with diminished cognition benefited more from the HF-DMP than patients with normal cognition, findings that were consistent considering at 30- and 90-day readmissions or death and also the difference in days at home at 1 year. In fact, patients with normal cognition did not seem to experience any benefit from this HF-DMP. Still, one should note that patients with moderate/severe cognitive impairment also lost some of the potential HF-DMP benefit at 90 days.

It should be highlighted that one of the particularities of this HF-DMP is that it was delivered during 30 days after hospital discharge with home visits and phone calls, that were repeated several times and involving family/carer whenever possible. This might have been a key to the success of this program compared with others that were applied only in the hospital. ${ }^{7}$ However, even with this 30-day extended intervention patients with normal cognitive function did not benefit from the intervention and patients with moderate/severe cognitive impairment only benefited during the 30 days of ongoing HF-DMP intervention. These findings suggest that there might exist a "sweet spot" of cognitive function where HF-DMP may provide the 
most benefit. It is possible that patients with normal cognition do not benefit form HFDMP because they can recall the information given during the hospital stay; on the other hand, patients with moderate/severe cognitive impairment only benefit during the intervention period and not after the intervention has stopped, because they may experience difficulties in recalling the instructions that had been given.

Based on the aforementioned findings, this study supports a personalized HF-DMP based on cognitive function screening, whereby patients with normal cognition would not be enrolled in such program, patients with mild cognitive impairment could benefit from a short- to medium-term intervention, and patients with moderate/severe cognitive impairment could benefit from continuous HF-DMP support to reduce readmissions and mortality. Figure 1. Of course, this hypothesis should be formally tested in a randomised controlled trial (RCT) before clinical application.

In conclusion, cognitive screening tools might help tailoring HF-DMP to patients' needs, but such hypothesis should be formally tested in a RCT.

\section{Disclosures}

None. 
Figure 1. Step-up support with HF-DMP based on cognitive function

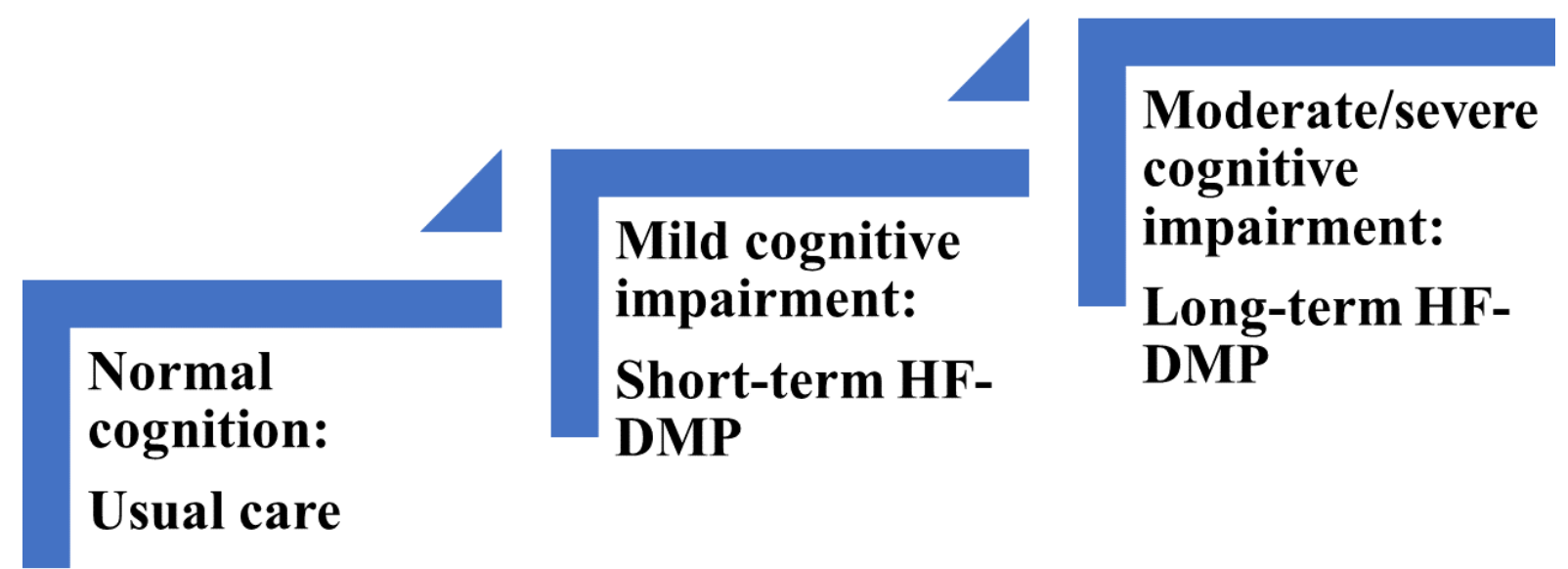

Legend: HF-DMP, heart failure disease management program. 


\section{References}

1. Cannon JA, Moffitt P, Perez-Moreno AC, et al. Cognitive Impairment and Heart Failure: Systematic Review and Meta-Analysis. J Card Fail. Jun 2017;23(6):464-475. doi:10.1016/j.cardfail.2017.04.007

2. Alves TC, Rays J, Fráguas R, Jr., et al. Localized cerebral blood flow reductions in patients with heart failure: a study using 99mTc-HMPAO SPECT. J Neuroimaging. Apr 2005;15(2):150-6. doi:10.1177/1051228404272880

3. Huynh Q, Negishi K, De Pasquale CG, et al. Validation of Predictive Score of 30-Day Hospital Readmission or Death in Patients With Heart Failure. Am J Cardiol. Feb 1 2018;121(3):322-329. doi:10.1016/j.amjcard.2017.10.031

4. Leto L, Feola M. Cognitive impairment in heart failure patients. J Geriatr Cardiol. Dec 2014;11(4):316-28. doi:10.11909/j.issn.1671-5411.2014.04.007

5. Holm H, Bachus E, Jujic A, et al. Cognitive test results are associated with mortality and rehospitalization in heart failure: Swedish prospective cohort study. ESC Heart Fail. Oct 2020;7(5):2948-2955. doi:10.1002/ehf2.12909

6. Amarasingham R, Patel PC, Toto K, et al. Allocating scarce resources in real-time to reduce heart failure readmissions: a prospective, controlled study. BMJ Qual Saf. Dec 2013;22(12):998-1005. doi:10.1136/bmjqs-2013-001901

7. Davis KK, Mintzer M, Dennison Himmelfarb CR, Hayat MJ, Rotman S, Allen J. Targeted intervention improves knowledge but not self-care or readmissions in heart failure patients with mild cognitive impairment. Eur J Heart Fail. Sep 2012;14(9):1041-9.

doi:10.1093/eurjhf/hfs096 